\title{
GENERAL THERMODYNAMICS.
}

Thermodynamics : An Introductory Treatise Dealing Mainly with First Principles and their Direct Applications. By G. H. Bryan. Teubner's Sammlung, Band XXI. Leipzig, 1907. xiv +204 pp.

The books on thermodynamics are legion. In purpose and content they run the whole gamut from Maxwell's inimitable little work on heat and the many no more advanced though greatly inferior treatments of the subject to the large treatises like Weinstein's Thermodynamik und Kinetik der Körper in three sizable and highly mathematical volumes of which the last is not yet finished. Some are chiefly concerned with engineering problems, others with physics, and lately many with physical chemistry. One might therefore well ask what was left for a new work on thermodynamics except repetition. Anybody who has undertaken to teach a comprehensive course on thermodynamics, beginning essentially at the foundations and covering as well as may be the various applications up to and even including those in physical chemistry, will have no hesitation in answering that there is no short treatise which he could use as a guide - as a précis - for his whole course, nothing that at once was comprehensive and compact, as deductive as might be and yet thoroughly physical, and furthermore well balanced between the distracting needs of the different dependent fields of science. Better might it be said that such would have been the answer a few months ago. Now it would no longer be true; for Bryan's little book furnishes precisely this desideratum.

It should be noted that there is no necessity of banishing the physical side of a science in giving a deductive presentation of its theory. The history of most branches of mathematical physics is about this: First some fundamental experiments combined with a very crude theory which is frequently almost as much wrong as right; then after an increasing accumulation of facts there appears some master mind to formulate a comprehensive and essentially correct theory based upon a few crucial experiments; finally the deductive stage arrives in which the start is made from certain mathematical statements and physical experiment no longer plays any considerable rôle. This 
last stage is, however, that in which physical interpretation should be most in evidence, and it is the omission of this constant a posteriori contact with nature that renders so much mathematical physics futile as far as physical science is concerned. The advance must not be away from physics and into mathematics; it should be from a priori physics and a posteriori mathematics to a priori mathematics and a posteriori physics. This last point of view is apparently that adopted by Bryan in regard to thermodynamics, as it is by most modern authors in regard to mechanics and the classical theories of optics.

The author divides his work into three parts: Physical aspects of thermodynamics, pages 1-26. The foundations of rational thermodynamics, pages 27-96. Thermodynamics of particular systems, pages 97-198.

From the title of the first part it might appear that we were still in the stage of a priori physics and close contact with experiment. A perusal of the text will rapidly disabuse one of any such notion. As a matter of fact only such reference to material bodies is made as is necessary for the formulation of the definitions and laws upon which thermodynamics is to be built. It would not do to say that in this first part the author lays down axioms for a thermodynamics; his procedure, subject to later revision, is analogous to Newton's in mechanics. Heat is taken as a fundamental idea just as force was. In fact as we find in mechanics the law that, when force acts, the amount of momentum is changed by the amount of the impulse of the force, so we find here the law that, when heat is transformed into work or conversely, the quantity of heat gained or lost is proportional to the quantity of work lost or gained. And there is the immediate additional statement that this law affords a measure of heat just as Newton's law gives a measure of force. Some might wish to banish heat from thermodynamics as they have banished force from dynamics. The attempt to do so does not seem particularly valuable from a pedagogic point of view, and it is probably well that Bryan is content at this early stage of his work to stay his desire for deduction just where he does. The concluding chapter of part I deals with the necessary changes of variable incident to the transformation of the many thermal coefficients previously defined.

Part II begins with an admirable discussion of various behaviors of energy - conservation, localization, reversibility, 
availability, and the dependence of the last on external conditions.* This might all find its way quite appropriately into many treatises on mechanics. The author then proceeds to discuss irreversibility with the definition that a change is said to be irreversible when the system cannot of itself change back to its original condition, and he lays down two axioms (from now on the word axiom is often in evidence): 1) If a system can undergo an irreversible change it will do so. 2) A perfectly reversible change cannot take place of itself; such a change can only be regarded as the limiting form of an irreversible change. It seems as though the second axiom more or less vitiates the immediately preceding definition of irreversibility. Although the difficulty is obviated before many pages have been turned, it might have been better to phrase the statements a little more carefully. The same may be said of the later sentence which expresses the fundamental principle of degradation : In all irreversible transformations available energy is lost, never gained. If anything in the way of emphasis is to be added to lost, it would be well to say something as definite as never gained or conserved. Lost and gained do not together form a universe of discourse in this case. These, however, are small matters to throw in the face of an author who is perhaps the first to have thoroughly conscientious scruples as regards definitions and axioms in his upbuilding of thermodynamics. $\dagger$

* The author's characterization of energy on page 39 is far from possessing the completeness and definiteness which is desirable in an entity that is to be made the basis of his whole deductive system. To critics inclined to be too severe at this point, may be replied with great reason that in the present state of our knowledge it is apparently impracticable to build up a perfectly definite system of rational thermodynamics. Indefiniteness is bound to occur somewhere unless the number of undefined elements is made larger than Bryan wishes to make it. To leave energy a somewhat vague and elastic conception is probably far better than to have some other entity obscure or undefined - and for precisely the reason that energy is so familiar and fundamental in all physics as to spare definition better than anything else. Furthermore, from a logical point of view, it should be noted that with the statement on page 39 must be included all the later axioms which go to contribute additional precision to the basic undefined symbol "energy."

+ It is only fair to mention at this point Perrin's Traité de chimique physique: les principes, Paris, Gauthier-Villars, 1903. Perrin is here writing for chemists. He goes so carefully into all the fundamental ideas of thermodynamics as to make his hook almost tedious reading for anybody who knows as much about energy and entropy as a chemist ought to ; but as the chemists do not know as much about such things as they should, the author is doubtless forced to follow the laborious detail that he does. Furthermore Perrin chooses to keep in much closer contact with experiment than Bryan and to 
After some further treatment of the characteristics of a system we call thermodynamic, there appears the definition of the quantity of heat involved in a thermodynamic transformation, namely,

$$
d Q=d U+d W
$$

where $d U$ is the increase of energy in the system and $d W$ the amount of external work done by the system. A discussion of the Carnot's cycle in its relation to the principle of the degradation of energy, which the author adopts as more fundamental than the usual statement of the second law, then leads to the definition of temperature and of absolute temperature (thermodynamic scale) in particular. The author's presentation of this whole introductory matter is certainly not hackneyed. It can only be characterized as original. It is extremely illuminating and peculiarly satisfactory to the mathematician who naturally demands closer formal reasoning than the physicist. It is to be hoped that future treatments of the subject will borrow much of the clearness and logic of Bryan's even in such frequent cases as those in which a complete adoption of his method would be inadvisable for one reason or another.

Thus far we have covered six chapters. The remaining chapters of this part may be spoken of more briefly. They deal with the usual topics. Chapter VII takes up the relation of available energy to entropy. Two definitions of entropy are given and each is elaborated. Homogeneous and heterogeneous systems are alike treated in detail, and so with both reversible and irreversible transformations. This chapter is long and clear; every reader has ample opportunity to become so thoroughly familiar with entropy as to forget that it ever was considered a weird bugaboo. The following two chapters deal with the matters of stability and thermodynamic potentials.

Part III is on the thermodynamics of particular systems. That the author should start off with the treatment of radiation is a bit of brave novelty. As a matter of fact such systems

depend less on rational dynamics; his work is not so completely deductive. This again is probably the wisest course for him to follow. To any who find difficulty in Bryan's Thermodynamics, especially in these underlying chapters, Perrin's Principes is heartily to be recommended; and even for those who find no trouble in following Bryan, Perrin has much of interest to offer. 
are perhaps the simplest, and the deduction of Stefan's law with the further proof that optical methods cannot increase availability gives to the subject at the very start an interest and vigor which the older treatments of perfect gases cannot approach. The succeeding chapter is largely a collection of formulas expressing the various thermal coefficients of simple systems in terms of the potentials. Then follows in chapter XII some account of gaseous systems whether pure or mixed. No very great detail is entered upon; true to the author's general intention the chapter is merely a sketch. Chapter XIII contains an account of the determination of thermodynamical quantities and will be especially welcome to those who like to see how the fundamental magnitudes are derivable from experimental data.

The next three chapters, XIV, XV, XVI, touch on the physico-chemical aspect of thermodynamics. The first takes up van der Waals's equation and the critical states, and also the phase rule and triple point for a single substance. The second treats the general question of phase equilibrium for heterogeneous substances. The third, which deals with reversible thermoelectric effects, gives Helmholtz's and Gibbs's formulas for reversible elements and an excellent discussion of the Kelvin and Peltier effects. In the next and last chapter are found some geometric and dynamical representations of thermodynamic phenomena. As might be expected these are for the most part Gibbs's surfaces and related diagrams on the one hand and the cyclic dynamical systems on the other. It seems as if it might have been well to add just a few words more on strictly statistical methods with perhaps a mention of the analogies to thermodynamics which Gibbs pointed out in his last work.* It should be borne in mind, however, that to say merely a few words which shall be comprehensible is a very difficult matter in a subject so technical and formulaic as statistical mechanics.

There remains merely a short conclusion, which again and for the last time emphasizes the fundamental points of the text. An elaborate index closes the volume. Although this is scarcely necessary in a work so concise and so well arranged as the present, it is surely not in the way.

From the foregoing sketch of the contents and general aim of Bryan's two hundred pages, it may be possible to gain some

* Elementary Principles in Statistical Mechanics, Charles Scribner's Sons, New York, 1907. 
insight into the character of the work, and to see that an empty place in our scientific literature has been discovered and satisfactorily filled. As was stated before, this book furnishes an excellent précis for anybody lecturing on general thermodynamics. If in addition there were in existence an elaborate treatise of three or four times the length following the same order of arrangement and amply supplied with physical applications and developments, the student of thermodynamics would scarcely have need of any lectures. As it is, both student and teacher are so much better off than they were a twelvemonth ago that it would be a bit ungracious already to ask for more - or would it be the highest expression of gratitude?

EDWIN BIDWELL Wilson.

Massachusetts Institute of Technology, Boston, September, 1907.

\section{SHORTER NOTICES.}

Funzioni poliedriche e modulari. By G. Vivanti. Milan, Hoepli, 1906. viii +437 pp.

THE object of this work cannot better be characterized than by the following quotation from the preface:

"The present volume has the modest scope of preparing the reader for the study of the classic lectures of Klein on the icosahedron and of the treatise on the elliptic modular functions by Klein and Fricke. The first of these, a model of elegance and a veritable mine of new and interesting ideas, is quite difficult to read both on account of the many concepts which are barely outlined and still more because, even after the various details have been understood, the connection which binds them, the guiding idea, is far from evident and comes to light only after a profound study and a thorough review of the entire material. The second work, on account of the mass and the multiformity of its contents, does not lend itself readily to an introductory study. On account of the lack of a suitable treatise for facilitating acquaintance with the theory of the polyhedral and modular functions, I believed it my duty to make available for others the work of elaboration which I have performed for my own use, thus saving them the repetition of this useful but laborious work. 